\title{
On the Cohen-Macaulayness of multi-Rees algebras and Rees algebras of powers of ideals
}

\author{
By Thomas Korb and Yukio NaKamura
}

(Received Sept. 26, 1995)

(Revised May 20, 1996)

\section{Introduction.}

Let $(A, \mathrm{~m})$ be a Noetherian local ring and $I$ an ideal in $A$. For a positive integer $r$, the $r$-th multi-Rees algebra of $A$ with respect to $I$ is defined as the $N_{0}^{r}$-graded ring:

$$
R_{A}\left(\mathbf{I}_{r}\right)=\bigoplus_{\underline{n} \geq \underline{0}} I^{n_{1}} \cdots I^{n_{r}}
$$

where $\underline{n}$ stands for an $r$-tuple $\left(n_{1}, \ldots, n_{r}\right)$ of natural numbers. Very often one identifies $R_{A}\left(\mathbf{I}_{r}\right)$ with $A\left[I t_{1}, I t_{2}, \ldots, I t_{r}\right] \subseteq A\left[t_{1}, t_{2}, \ldots, t_{r}\right]$ where $t_{1}, t_{2}, \ldots, t_{r}$ are indeterminates over $A$. The notation $\mathbf{I}_{r}$ should be considered as the $r$-tuple $(I, \ldots, I)$. In particular, in case $r=1$, the multi-Rees algebra is nothing else but the (ordinary) Rees algebra which we denote by $R_{A}(I)$. For the following, we denote the maximal homogeneous ideal of $R_{A}(I)$ by $M$.

The concept of multi-Rees algebras is e.g. connected with mixed multiplicities (cf. [Te] and [HHRTa]) and joint reductions (cf. [Re]). Verma studied for instance the Cohen-Macaulayness of multi-Rees algebras of ideals having joint reduction number zero (see [Ve]). He considered multi-Rees algebras with respect to different ideals $I_{1}, \ldots, I_{r}$ of $A$, i.e. $A$-algebras of the form: $A\left[I_{1} t_{1}, I_{2} t_{2}, \ldots, I_{r} t_{r}\right]$, but only in the case that $\operatorname{dim} A=2$ (plus some additional assumptions). A result on the Cohen-Macaulayness of multi-Rees algebras of m-primary ideals in a local ring $A$ of dimension two having joint reduction number zero can also be found in [HHRTa]. From a totally different viewpoint, Goto and Nishida studied the Cohen-Macaulay and Gorenstein property of $R_{A}\left(\mathbf{I}_{2}\right)$ in their work on Rees algebras defined by a filtration ([GN]). A detailed characterization of the Cohen-Macaulay and Gorenstein property of multi-Rees algebras was given by Herrmann, Hyry and Ribbe (et al.) in [HHR1], [HHR2] and [HHRTa]. (Their results do not only deal with properties of $R_{A}\left(\mathbf{I}_{r}\right)$, but also with properties of multi-Rees algebras of the form $A\left[I^{k_{1}} t_{1}, \ldots, I^{k_{r}} t_{r}\right]$, where $k_{1}, \ldots, k_{r}$ are positive integers.) Some newer results on the Cohen-Macaulayness of multi-Rees algebras and its relation with filter-regularity in the corresponding form ring can be found in [HHK].

One of the most useful results on the Cohen-Macaulay property of multiRees algebras, which we will also frequently use throughout this paper, is (cf. [HHR1, 2.2]): 
THEOREM 1.1 (Herrmann/Hyry/Ribbe). Let $(A, \mathfrak{m})$ be a local ring of dimension $d$ and I an ideal in $A$ of positive height. Then the following conditions are equivalent for $a$ positive integer $r$ :

(a) $R_{A}\left(\mathbf{I}_{r}\right)$ is a Cohen-Macaulay ring.

(b) $\left[H_{M}^{i}\left(R_{A}(I)\right)\right]_{n}=(0)$ for all $i<d+1$ and $n \notin\{-r+1, \ldots,-1\}$.

(c) $\left[H_{M}^{i}\left(G_{A}(I)\right)\right]_{n}=(0)$ for all $i<d$ and $n \notin\{-r, \ldots,-1\}$, and $a\left(G_{A}(I)\right)<0$.

Here, we denoted the factor ring $R_{A}(I) / I R_{A}(I)$ by $G_{A}(I)$. This is the so called form ring (or: associated graded ring) of $A$ with respect to $I$. For the $i$-th graded local cohomology with respect to $M$ we use, as usual, the notation $H_{M}^{i}\left({ }_{-}\right)$. And following $[\mathbf{G W}]$ we put $a\left(G_{A}(I)\right):=\sup \left\{n \in Z \mid\left[H_{M}^{d}\left(G_{A}(I)\right)\right]_{n} \neq(0)\right\}$ and call it the $a$-invariant of $G_{A}(I)$.

In the case $r=1$, the equivalence in the above theorem is nothing else but the characterization of the Cohen-Macaulayness of the Rees algebra $R_{A}(I)$ as given by Trung and Ikeda in [TI]. So the theorem shows that multi-Rees algebras behave to some extend as ordinary Rees algebras with respect to the Cohen-Macaulay property (since this can be described in terms of properties of the local cohomology modules of $R_{A}(I)$ resp. $\left.G_{A}(I)\right)$.

Theorem 1.1 also shows immediately that the Cohen-Macaulayness of $R_{A}\left(\mathbf{I}_{r}\right)$ implies the same property for $R_{A}\left(\mathbf{I}_{s}\right)$ and $R_{A}\left(I^{s}\right)$ for all $s \geq r$. An important special case of the first of these two implications is the case $r=1$, i.e. that if the Rees algebra of an ideal is Cohen-Macaulay, the same holds for all corresponding multi-Rees algebras. For the second implication recall that $R_{A}\left(I^{s}\right)$ coincides with the $s$-th Veronese subring $R_{A}(I)^{(s)}$ of $R_{A}(I)$ as graded algebras. Hence we have isomorphisms $\left[H_{M}^{i}\left(R_{A}(I)\right)\right]_{n s} \cong$ $\left[H_{M^{\prime}}^{i}\left(R_{A}\left(I^{s}\right)\right)\right]_{n}$ for all $n \in Z$, where $M^{\prime}$ is the maximal homogeneous ideal of $R_{A}\left(I^{s}\right)$.

On the other hand, it is known that the Cohen-Macaulayness of $R_{A}\left(I^{s}\right)$ for some $s \geq 2$ does not have to imply that $R_{A}\left(\mathbf{I}_{r}\right)$ is Cohen-Macaulay for some $r \geq 1$ (see Example 2.6).

In [HRZ, 2.7] one can find the result that for ideals $I$ of positive height the CohenMacaulay property of $R_{A}\left(I^{s}\right)$ for some $s \in N$ is equivalent to the Cohen-Macaulayness of $R_{A}(I)$ if $G_{A}(I)$ is Cohen-Macaulay. Observe that the corresponding statement for multi-Rees algebras (i.e. replace $R_{A}\left(I^{s}\right)$ by $R_{A}\left(\mathbf{I}_{s}\right)$ in the above result) is an immediate consequence of Theorem 1.1.

These observations lead to the following two questions which we will address in this work:

I. How are the Cohen-Macaulay properties of $R_{A}\left(I^{s}\right)$ and $R_{A}\left(\mathbf{I}_{r}\right)$ related?

II. In which situations does the Cohen-Macaulayness of one of the above Rees algebras already imply the Cohen-Macaulayness of $R_{A}(I)$ ?

To explain our results, we need one more notation; we put:

$$
a_{i}:=\sup \left\{n \in Z \mid\left[H_{M}^{i}\left(G_{A}(I)\right)\right]_{n} \neq(0)\right\}
$$

and call $a_{i}$ the $i$-th a-invariant (or: $a_{i}$-invariant) of $G_{A}(I)$. Since $\operatorname{dim} G_{A}(I)=d$, we 
have by definition that $a_{d}=a\left(G_{A}(I)\right)$, where the right side in this equation is the $a$-invariant which we introduced above.

Our main result with respect to question $I$ can now be stated as:

THEOREM 1.2. Let $(A, \mathrm{~m})$ be a Noetherian local ring of dimension $d$ and $I$ an ideal in $A$ of positive height. Let $M$ be the maximal homogeneous ideal of $R_{A}(I)$ and suppose that depth $G_{A}(I)_{M} \geq d-1$. Then the following conditions are equivalent:

(a) $R_{A}\left(I^{s}\right)$ is a Cohen-Macaulay ring for some $s>0$.

(b) $R_{A}\left(\mathbf{I}_{r}\right)$ is a Cohen-Macaulay ring for some $r>0$.

In general, we can not expect a generalization of Theorem 1.2 to the case that depth $G_{A}(I)_{M} \leq d-2$. In fact, Example 2.6 will tell us that there exists an m-primary ideal in a 2-dimensional local domain such that $R_{A}\left(I^{s}\right)$ is Cohen-Macaulay for all $s \geq 2$, but $R_{A}\left(\mathbf{I}_{r}\right)$ is not Cohen-Macaulay for all $r \geq 1$. However, even if depth $G_{A}(I)_{M} \leq$ $d-2$, we can have the following Proposition 1.3. Indeed, Theorem 1.2 immediately follows from Proposition 1.3, because the condition (ii) in Proposition 1.3 always holds if depth $G_{A}(I)_{M} \geq d-1$.

Proposition 1.3. Let $A$ and $I$ be as in Theorem 1.2 and put $g:=\operatorname{depth} G_{A}(I)_{M}$. Suppose that one of the following conditions holds:

(i) $a_{g+2 i}<0$ for all $i \geq 0$ with $2 i<d-g$,

(ii) $a_{g+2 i+1}<0$ for all $i \geq 0$ with $2 i+1<d-g$.

Then the following statements are equivalent:

(a) $R_{A}\left(I^{s}\right)$ is a Cohen-Macaulay ring for some $s>0$.

(b) $R_{A}\left(\mathbf{I}_{r}\right)$ is a Cohen-Macaulay ring for some $r>0$.

To investigate question II, we concentrate on the case that $A$ is a Cohen-Macaulay local ring of dimension at most three. Our main results are as follows:

THEOREM 1.4. Let $A$ be a Cohen-Macaulay local ring of dimension 2 and I an ideal of positive height. Then the following conditions are equivalent:

(a) $R_{A}\left(\mathbf{I}_{r}\right)$ is a Cohen-Macaulay ring for some $r>0$.

(b) $R_{A}(I)$ is a Cohen-Macaulay ring.

THEOREM 1.5. Let I be an ideal in a Cohen-Macaulay local ring $A$ of dimension 3. Suppose that $I$ is either an integrally closed m-primary ideal, or $A / I$ is a 1dimensional Cohen-Macaulay ring. Then the following conditions are equivalent:

(a) $R_{A}\left(\mathbf{I}_{r}\right)$ is a Cohen-Macaulay ring for some $r>0$.

(b) $R_{A}(I)$ is a Cohen-Macaulay ring.

Theorem 1.4 is an expansion of [HHRTa, 2.4], where the same was proven for m-primary ideals. Theorem 1.5 was also motivated by [HHRTa] where it was shown that the Cohen-Macaulayness of a multi-Rees algebra of the maximal ideal $m$ of $a$ 3-dimensional Cohen-Macaulay local ring implies the same property for $R_{A}(\mathrm{~m})$.

In the appendix of [HHR2], Trung gave an ideal $I$ in a non-Cohen-Macaulay Buchsbaum local domain $A$ of dimension 3 such that $R_{A}(I)$ is not Cohen-Macaulay, 
but $R_{A}\left(\mathbf{I}_{2}\right)$ is a Cohen-Macaulay (even: Gorenstein) ring. (See also Example 2.14.) So we can not expect that the implication in question II holds in general. But motivated by the above mentioned results, one might ask the following (cp. also Remark 2.13):

Let $A$ be a Cohen-Macaulay local ring and $I$ an ideal of positive height. Suppose $R_{A}\left(\mathbf{I}_{r}\right)$ is a Cohen-Macaulay ring for some $r \geq 2$. Does it then follow (maybe under some assumptions) that already $R_{A}(I)$ is Cohen-Macaulay?

Up to now, it is not clear how to answer this question. If the implication in the question does not hold in general, it would be very interesting for us to see counterexamples. By the result in Theorem 1.2 it would be enough to find an ideal $I$ of positive height in a Cohen-Macaulay local ring such that depth $G_{A}(I)_{M}=d-1, R_{A}(I)$ is not Cohen-Macaulay, but $R_{A}\left(I^{s}\right)$ is Cohen-Macaulay for some $s \geq 2$.

Maybe the most important special case which is still open with respect to the above question is that of m-primary ideals in a Cohen-Macaulay local ring $A$ of dimension three which are not integrally closed. Assume that $I$ is such an ideal and that the above question has a negative answer for $I$. Then one can show, using results on filterregularity in $G_{A}(I)$ from $[\mathbf{H H K}]$ and our Lemma 3.1, that the following must hold: $a_{0}=a_{1}=-\infty, a_{2}=-2$ and $a(G)=-1$. In this case, the reduction exponent of $I$ is 2.

From a different point of view, Remark 2.8 is also related to the above question, and the authors believe that investigations in this direction might be fruitful, too.

We close this section with a view of this paper. We will prove Proposition 1.3 and state some related results in Section 2. E.g., as already mentioned, it follows from Theorem 1.1 that $R_{A}\left(I^{s}\right)$ is Cohen-Macaulay for all $s \geq r$ once $R_{A}\left(\mathbf{I}_{r}\right)$ is so. Therefore one might reformulate question $I$ in a stronger way, i.e.: Does the CohenMacaulayness of $R_{A}\left(I^{s}\right)$ imply the same property for $R_{A}\left(\mathbf{I}_{s}\right)$ ? (cf. Some answers to this question will be given in Theorem 2.12. Section 3 is devoted to the proofs of Theorem 1.4 and Theorem 1.5 and some additional results.

Throughout this paper, $(A, \mathfrak{m})$ is a Noetherian local ring of dimension $d$ and $I$ is an ideal in $A$. For short, we put $R:=R_{A}(I)$ and $G:=G_{A}(I)$ and denote the maximal homogeneous ideal of $R$ by $M$. Very often, we will identify $R$ with $A[I t]$, where $t$ is an indeterminate over $A . \quad H_{\mathfrak{m}}^{i}\left({ }_{-}\right)$(resp. $\left.H_{M}^{i}()_{-}\right)$) stands for the $i$-th local cohomology functor with respect to $\mathrm{m}$ (resp. $M$ ) in the category of $A$-modules (resp. graded $R$ modules). For information on graded local cohomology we refer to [HIO, Chap. VII].

\section{Proof of Proposition 1.3.}

Let $\left(S_{0}, \mathfrak{n}\right)$ be a Noetherian local ring and $S$ a standard graded algebra over $S_{0}$, that is a Noetherian graded algebra of the form $S=S_{0}\left[S_{1}\right]$. Let $N$ be the maximal homogeneous ideal of $S$ and $a$ a homogeneous ideal in $S$. We say that $S$ is a generalized Cohen-Macaulay ring with respect to $\mathfrak{a}$ (for short: genCM w.r.t. $\mathfrak{a}$ ), if $\mathfrak{a}^{n} H_{N}^{i}(S)=(0)$ for all $n \gg 0$ and all $i<\operatorname{dim} S$. In particular, the fact that $S$ is genCM w.r.t. $S_{+}\left(S_{+}:=\bigoplus_{n>0} S_{n}\right)$ is equivalent to $\left[H_{N}^{i}(S)\right]_{n}=(0)$ for all $n \ll 0$ and $i<\operatorname{dim} S$ by 
[TI, 2.2]. Although the next lemma is known, we give a short proof for the reader's convenience:

LEMMA 2.1. Suppose that $S_{0}$ is a homomorphic image of a Gorenstein local ring and $S_{N}$ is equidimensional. Then the following conditions are equivalent:

(a) $S$ is a genCM ring w.r.t. $S_{+}$.

(b) $\operatorname{Proj}(S)$ is a Cohen-Macaulay scheme.

Proof. Since $S$ is generated by elements of degree 1 , saying that $\operatorname{Proj}(S)$ is a Cohen-Macaulay scheme is equivalent to saying that $S_{Q}$ is a Cohen-Macaulay ring for all $Q \in \operatorname{Proj}(S)$ (cf. e.g. [HIO, §12]). On the other hand, $S_{N}$ is equidimensional and catenary, hence we have $\operatorname{dim} S_{N} / Q+\operatorname{dim}\left(S_{N}\right)_{Q}=\operatorname{dim} S_{N}$ for all $Q \in \operatorname{Spec}\left(S_{N}\right)$. This implies, in particular, $\operatorname{dim} S / Q+\operatorname{dim} S_{Q}=\operatorname{dim} S$ for all $Q \in \operatorname{Proj}(S)$. Thus the stated equivalence of the lemma immediately follows from [HIO, (43.3)].

We fix the following notation for the $a$-invariants and $e$-invariants, which we will use frequently throughout this paper:

Definition 2.2. Let $E$ be a finitely generated graded $S$-module. Then we define for all $i \in Z$ the $i$-th $a$-invariant (resp. $e$-invariant) of $E$ with respect to $N$ as:

$$
\begin{aligned}
& a_{N}^{i}(E):=\sup \left\{n \in Z \mid\left[H_{N}^{i}(E)\right]_{n} \neq(0)\right\} \\
& e_{N}^{i}(E):=\inf \left\{n \in Z \mid\left[H_{N}^{i}(E)\right]_{n} \neq(0)\right\}
\end{aligned}
$$

According to our definitions earlier in this paper, we use the shorter notations: $a_{i}:=a_{M}^{i}(G), e_{i}:=e_{M}^{i}(G)$ and $a(G):=a_{M}^{d}(G)$. (If there is confusion possible, we will use the longer notation $a_{i}(G)$ instead of $a_{i}$.)

It is well known that we always have in our setting: $a_{N}^{i}(E) \neq \infty . \quad$ If $H_{N}^{i}(E)=(0)$, we have by definition: $a_{N}^{i}(E)=-\infty$ and $e_{N}^{i}(E)=\infty$. If $S$ is genCM w.r.t. $S_{+}$, both $a_{N}^{i}(S)$ and $e_{N}^{i}(S)$ are finite for $i<\operatorname{dim} S$ (unless $H_{N}^{i}(S)=(0)$ ) by the above characterization of the generalized Cohen-Macaulay property.

Let $\hat{S}_{0}$ be the $n$-adic completion. Then it holds that $H_{N}^{i}(S) \otimes_{S_{0}} \hat{S}_{0} \cong H_{\hat{N}}^{i}(\hat{S})$, where $\hat{S}=S \otimes_{S_{0}} \hat{S}_{0}$ and $\hat{N}=N \otimes_{S_{0}} \hat{S}_{0}$. Thus saying that $S$ is genCM w.r.t. $S_{+}$is equivalent to saying that $\hat{S}$ is genCM w.r.t. $\hat{S}_{+}$. For a finitely generated graded $S$-module $E$, we denote the depth of $E_{N}$ over the local ring $S_{N}$ by depth $E$.

For the rest of this section, we assume that the ideal $I \subseteq A$ has positive height. In particular, we know in this case that $\operatorname{dim} R=d+1$.

We will frequently make use of the following two short exact sequences which were introduced by Huneke in $[\mathbf{H u}]$. Here, $R_{+}$denotes the irrelevant ideal of $R$.

$$
\begin{gathered}
0 \longrightarrow R_{+} \longrightarrow R \longrightarrow A \longrightarrow 0 \\
0 \longrightarrow R_{+}(1) \longrightarrow R \longrightarrow G \longrightarrow 0
\end{gathered}
$$

Lemma 2.3. Suppose that $R_{A}\left(I^{s}\right)$ is Cohen-Macaulay for some $s>0$. Then the following holds: 
(a) $a(G)<0$.

(b) $\left[H_{M}^{i}(R)\right]_{0}=(0)$ for all $i \in \boldsymbol{Z}$.

Proof. Since $R_{A}\left(I^{s}\right)$ is Cohen-Macaulay, we get from [TI, 1.1]: $a\left(G_{A}\left(I^{s}\right)\right)<0$. Knowing this, the following formula from [HRZ] (which holds in our setting by [HZ, 4.2]) shows (a):

$$
a\left(G_{A}\left(I^{s}\right)\right)=\left[\frac{a(G)}{s}\right] \quad(\text { Here },[\cdot] \text { denotes the integral part. })
$$

Let $M^{\prime}:=M \cap R_{A}\left(I^{s}\right)$, which is the maximal homogeneous ideal of $R_{A}\left(I^{s}\right)$. Since $R_{A}\left(I^{s}\right) \cong R^{(s)}$, we then have:

$$
\left[H_{M^{\prime}}^{i}\left(R_{A}\left(I^{s}\right)\right)\right]_{n} \cong\left[H_{M}^{i}(R)^{(s)}\right]_{n}=\left[H_{M}^{i}(R)\right]_{n \cdot s}
$$

(cf. [GW, (3.1.1)]. Thus $\left[H_{M}^{i}(R)\right]_{n \cdot s}=(0)$ for all $i \leq d$ and $n \in Z$ since $R_{A}\left(I^{s}\right)$ is CohenMacaulay. The remaining case $i=d+1$ follows from the fact, that we always have: $a_{M}^{i}(R)=-1$ (cf. e.g. [GN, Part I (6.3)]).

Lemma 2.4. Suppose that $R_{A}\left(I^{s}\right)$ is Cohen-Macaulay for some $s>0$. Then we have for $i \in Z$ :

(a) If $a_{i-1}<0$, then $\left[H_{M}^{i}(R)\right]_{n}=(0)$ for all $n \geq 0$.

(b) If $a_{i-1}<0$ and if $a_{i} \geq 0$, then $a_{i}<a_{i+1}$.

Proof. Observe first, that we always get from sequence $(S 1):\left[H_{M}^{i}\left(R_{+}\right)\right]_{n} \cong$ $\left[H_{M}^{i}(R)\right]_{n}$ for $n \neq 0$. Now, since $a_{i-1}<0$, we get from sequence $(S 2)$ for $n \geq 0$ :

$$
0 \longrightarrow\left[H_{M}^{i}(R)\right]_{n+1} \longrightarrow\left[H_{M}^{i}(R)\right]_{n}
$$

from which we see, that $\left[H_{M}^{i}(R)\right]_{n}=(0)$ for $n \geq 0$. (Otherwise we would get by the above injective map that $\left[H_{M}^{i}(R)\right]_{0} \neq(0)$, which is a contradiction to Lemma 2.3 (b).) Hence assertion (a) follows.

So we get, by applying local cohomology functors to $(S 2)$, for $n \geq 0$ :

$$
0 \longrightarrow\left[H_{M}^{i}(G)\right]_{n} \longrightarrow\left[H_{M}^{i+1}(R)\right]_{n+1} \longrightarrow\left[H_{M}^{i+1}(R)\right]_{n} \longrightarrow\left[H_{M}^{i+1}(G)\right]_{n}
$$

from which we see immediately:

$$
\left[H_{M}^{i+1}(R)\right]_{a_{i}+1} \neq(0)
$$

In order to show assertion (b), we assume that $a_{i} \geq a_{i+1}$, i.e. $\left[H_{M}^{i+1}(G)\right]_{n}=(0)$ for $n \geq a_{i}+1$. Then we get the following isomorphisms from the above sequence: $\left[H_{M}^{i+1}(R)\right]_{n+1} \cong\left[H_{M}^{i+1}(R)\right]_{n}$ for $n \geq a_{i}+1$, which together with (1) imply: $\left[H_{M}^{i+1}(R)\right]_{n} \neq$ (0) for all $n \geq a_{i}+1$. But this is a contradiction and therefore we must have: $a_{i}<a_{i+1}$.

Proposition 2.5. The following two conditions are equivalent:

(a) $R_{A}\left(I^{s}\right)$ is a Cohen-Macaulay ring for some $s>0$.

(b) $R$ is a genCM ring w.r.t. $R_{+}$and $\left[H_{M}^{i}(R)\right]_{0}=(0)$ for all $i \in Z$. 
Proof. (b) $\Rightarrow\left(\right.$ a): Take an integer $s$ such that $s>\max \left\{\left|a_{M}^{i}(R)\right|,\left|e_{M}^{i}(R)\right|\right\}$ for all $i<\operatorname{dim} R$ with $H_{M}^{i}(R) \neq(0)$. This is possible by [TI, 2.2] (see our remarks after Definition 2.2). Using the isomorphism $\left[H_{M^{\prime}}^{i}\left(R_{A}\left(I^{s}\right)\right)\right]_{n} \cong\left[H_{M}^{i}(R)\right]_{n \cdot s}$, where $M^{\prime}=M \cap R_{A}\left(I^{s}\right)$, implies now that $R_{A}\left(I^{s}\right)$ is a Cohen-Macaulay ring as claimed. (a) $\Rightarrow(\mathrm{b})$ : We may assume that $A$ is complete. (See the part below Definition 2.2.) Suppose that $R_{A}\left(I^{s}\right)$ is Cohen-Macaulay. Then by [HIO, (18.23)], $A$ is equidimensional, and hence it follows from [HIO, (18.23)] again that $R_{M}$ is equidimensional. On the other hand, $\operatorname{Proj}(R)$ is a Cohen-Macaulay scheme since $\operatorname{Proj}(R) \cong$ $\operatorname{Proj}\left(R_{A}\left(I^{s}\right)\right)$. Hence by Lemma $2.1, R$ is genCM w.r.t. $R_{+}$. The rest of the proof now follows from Lemma 2.3 (b).

Proof of Proposition 1.3. The implication from (b) to (a) holds without any assumptions by Theorem 1.1. Therefore we just have to show the other direction. From Lemma 2.3 (a) we get $a(G)<0$. Together with this, one of the two assumptions of the theorem is sufficient to see by Lemma 2.4 (b) that all $i$-th $a$-invariants of $G$ are negative $($ or $-\infty)$. Observe, that $a_{0}, \ldots, a_{g-1}=-\infty$ by the characterization of depth via local cohomology. We want to apply Theorem 1.1 to see that $R_{A}\left(\mathbf{I}_{r}\right)$ is CohenMacaulay for some $r>0$. In order to do this, it just remains to show that: $\left[H_{M}^{i}(G)\right]_{n}=(0)$ for all $n \ll 0$ and all $i<d$. But this we can see from the CohenMacaulayness of $R_{A}\left(I^{s}\right)$ as follows: From Proposition 2.5 we get that $R$ is genCM w.r.t. $R_{+}$. By $[\mathbf{T I}, 2.2]$ this implies: $\left[H_{M}^{i}(R)\right]_{n}=(0)$ for all $n \ll 0$ and all $i<$ $d+1$. Using sequence $(S 2)$, we see that we then also have: $\left[H_{M}^{i}(G)\right]_{n}=(0)$ for all $n \ll 0$ and all $i<d$. This was to show.

REMARK. Some of the auxiliary results which we used to prove Proposition 1.3 are stated in [HZ, 4.7 (ii)]. But the result there (though stated for arbitrary ideals) and its proof (which refers to [Ho1]) only hold for the m-primary case (in the preprint-version of that paper). Therefore we did not refer to [HZ, 4.7 (ii)], but gave new proofs for everything we needed to show Proposition 1.3. This was communicated to the authors of $[\mathrm{HZ}]$ by the first author of the present work.

The following example shows that it is not possible to improve Theorem 1.2 (i.e. the statement of the theorem does not have to hold if depth $G<d-1$ ).

EXAMPLE 2.6. Let $B=k[[X, Y, Z]]$ be the formal power series ring over a field $k$. Let $A=B /\left(X Y-Z^{2}\right) B$ and $I=\left(X^{2}, Y^{2}, X Z, Y Z\right) A$. Then $R_{A}\left(I^{s}\right)$ is a CohenMacaulay ring for all $s \geq 2$, but $R_{A}\left(\mathbf{I}_{r}\right)$ is not Cohen-Macaulay for any $r \geq 1$.

Proof. Let $L=\left(X^{2}, Y^{2}, X Z, Y Z, Z^{2}\right) A$ and $J=\left(X^{2}, Y^{2}\right) A$. Then we can check that $L^{2}=J L, I \neq L$ and $Z^{2} I \subseteq I^{2}$. Hence $R_{A}(L)$ is a Cohen-Macaulay ring of dimension 3 (cf $[\mathbf{V V}, 3.1])$. Note that $L^{2}=I L$. Let $n \geq 2$. Then

$$
L^{n}=L I^{n-1}=I^{n}+Z^{2} I^{n-1}=I^{n} .
$$


Consider the following exact sequence:

$$
0 \longrightarrow R \longrightarrow R_{A}(L) \longrightarrow C \longrightarrow 0
$$

where $R=R_{A}(I)$ and $C=\bigoplus_{n \geq 0} L^{n+1} / I^{n+1}$. We observe that $C$ is concentrated in degree 1 and has dimension 0 as an $R$-module. Therefore we get from the above sequence:

$$
H_{M}^{i}(R) \cong \begin{cases}(0) & i=0 \\ {\left[H_{M}^{i}(R)\right]_{1} \cong L / I} & i=1 \\ (0) & i=2\end{cases}
$$

Hence by Theorem 1.1 and the isomorphism $\left[H_{M^{\prime}}^{i}\left(R_{A}\left(I^{S}\right)\right)\right]_{n} \cong\left[H_{M}^{i}(R)\right]_{n \cdot s}$, we obtain the statement of the example.

Another example where all $R_{A}\left(I^{s}\right)$ are Cohen-Macaulay for $s \geq 2$, but no corresponding multi-Rees algebra is Cohen-Macaulay, was constructed by Schenzel and can e.g. be found in [HRS]. And in [Ho1, 3.6], Hoa also gives an example which is similar to the one above, though his ring is not a hypersurface. (Hoa does not consider multiRees algebras. But he gives an explicit description of the local cohomology of the corresponding form ring which can be used in Theorem 1.1 to see that his example belongs to the class of examples described here.)

Next, we will describe the situation that $\operatorname{dim} A=1$.

Remark 2.7. Suppose that $\operatorname{dim} A=1$ and $I$ is m-primary. Then the CohenMacaulayness of $R_{A}\left(I^{s}\right)$ for some $s>0$ implies by [HIO, (45.4)] that $A$ is CohenMacaulay and $I^{s}$ is principal. But then $I$ must be principal, too (by [Sa, Prop. 1]). This yields that the following conditions are equivalent:

(a) $R_{A}\left(I^{s}\right)$ is a Cohen-Macaulay ring for some $s>0$.

(b) $R_{A}\left(\mathbf{I}_{r}\right)$ is a Cohen-Macaulay ring for some $r>0$.

(c) $R$ is a Cohen-Macaulay ring.

(b) $G$ is a Cohen-Macaulay ring and $a(G)<0$.

(See also Corollary 3.2.)

All of Remark 2.7 above also follows from Theorem 1.2 together with Theorem 1.1 and [TI, 1.1], observing that $G$ is Cohen-Macaulay and that the reduction exponent of $I$ is zero in this case.

In the next section we will prove other results on how the Cohen-Macaulay properties of $R_{A}\left(\mathbf{I}_{r}\right)$ and $R_{A}(I)$ are related. But we want to mention here one more known result (due to Ooishi) for the cases that $\operatorname{dim} A=2$ or 3 , though it needs a Gorenstein property in its assumptions:

REMARK 2.8. Let $I$ be an m-primary ideal in a Cohen-Macaulay local ring $A$ of dimension 2 or 3 . Suppose that $R_{A}\left(I^{s}\right)$ is a Gorenstein ring for some $s>0$. Then $R$ is Cohen-Macaulay. 
This follows from [Oo, 4.3], using the fact that $s \leq d-1$ (cp. [HRZ, 4.1] or [Oo, 4.1]).

Our next target in this section is to find relations between the smallest numbers $s$ and $r$ such that $R_{A}\left(I^{s}\right)$ and $R_{A}\left(\mathbf{I}_{r}\right)$ can be Cohen-Macaulay. Recall that we always have: $s \leq r$. In Theorem 2.12 we will sum up some conditions which guarantee the equality $s=r$ under the assumption that depth $G \geq d-1$. But first, we need some Lemmata. In the proofs of the following statements, we will frequently make use of the fact that:

$$
\left[H_{M}^{i}\left(R_{+}\right)\right]_{n} \cong\left[H_{M}^{i}(R)\right]_{n} \text { for } n \neq 0,
$$

which follows from sequence $(S 1)$, without mentioning it explicitly.

Lemma 2.9. Suppose that $R_{A}\left(I^{s}\right)$ is Cohen-Macaulay for some $s>0$. Then we have the inequality: $\operatorname{depth} R \geq \operatorname{depth} G+1$.

Proof. Let $i \leq \operatorname{depth} G$. From the sequences $(S 1)$ and $(S 2)$ we get the following injective maps for all $n \leq-2$ :

$$
0 \longrightarrow\left[H_{M}^{i}(R)\right]_{n+1} \longrightarrow\left[H_{M}^{i}(R)\right]_{n}
$$

Hence: $\left[H_{M}^{i}(R)\right]_{n}=(0)$ for all $n \leq-1$ since the Cohen-Macaulayness of $R_{A}\left(I^{s}\right)$ implies $\left[H_{M}^{i}(R)\right]_{m \cdot s}=(0)$ for all $m \in Z$ as we have already seen in the proof of Lemma 2.3. But since $a_{i-1}=-\infty$, we now get by Lemma $2.4(\mathrm{a}): H_{M}^{i}(R)=(0)$.

Lemma 2.10. Suppose that $R_{A}\left(I^{s}\right)$ is Cohen-Macaulay for some $s>0$. Assume moreover that $\operatorname{depth} A=\operatorname{depth} G=: g$. Then the following holds:

$$
\text { If } a_{g} \leq-2, \text { then }\left[H_{M}^{g+1}(R)\right]_{n} \neq(0) \text { for all } a_{g}+1 \leq n \leq-1 .
$$

In particular, we have: $s \geq-a_{g}$.

Proof. Observe first that depth $R \geq g+1$ by Lemma 2.9. Therefore we get from the long exact sequence of local cohomology coming from sequence $(S 1)$ :

$$
\left[H_{M}^{g+1}\left(R_{+}\right)\right]_{0} \neq(0) \text {. }
$$

Now, the injective maps:

$$
0 \longrightarrow\left[H_{M}^{g+1}\left(R_{+}\right)\right]_{n+1} \longrightarrow\left[H_{M}^{g+1}(R)\right]_{n} \quad\left(n \geq a_{g}+1\right),
$$

which we get from sequence $(S 2)$ imply together with (2) the statement of the lemma.

Lemma 2.11. Suppose that $R_{A}\left(I^{s}\right)$ is Cohen-Macaulay for some $s>0$. Then we have: $\quad \operatorname{depth} A \geq \operatorname{depth} G$.

Proof. Put $f:=\operatorname{depth} A$. Then we get from sequence $(S 1)$ together with Lemma 2.3 (b) that:

$$
\left[H_{M}^{f+1}\left(R_{+}\right)\right]_{0} \neq(0)
$$


Assume now: $\operatorname{depth} G>\operatorname{depth} A$. Then we have $H_{M}^{f}(G)=(0)$ and get so from sequence $(S 2)$ the following maps:

$$
0 \longrightarrow\left[H_{M}^{f+1}\left(R_{+}\right)\right]_{n+1} \longrightarrow\left[H_{M}^{f+1}(R)\right]_{n}
$$

If (3) holds, we can use the maps (4) to see that $\left[H_{M}^{f+1}(R)\right]_{n} \neq(0)$ for all $n \ll 0$. But this is a contradiction to Proposition 2.5 which says that the $i$-th local cohomology of $R$ is concentrated in finitely many degrees for $i \leq d$.

THEOREM 2.12. Suppose that depth $G \geq d-1$ and that one of the following conditions holds:

(i) $a(G) \leq e_{d-1}$.

(ii) $a(G) \leq a_{d-1}$ and $\left[H_{M}^{d-1}(G)\right]_{n} \neq(0)$ for all $e_{d-1} \leq n \leq a_{d-1}$.

(iii) $A$ is not Cohen-Macaulay and $\left[H_{M}^{d-1}(G)\right]_{n} \neq(0)$ for all $e_{d-1} \leq n \leq a_{d-1}$.

(iv) $e_{d-1} \geq-2$.

(v) $e_{d-1} \leq-3$ and $\left[H_{M}^{d-1}(G)\right]_{n} \neq(0)$ for all $e_{d-1} \leq n \leq-3$.

Then, for any $s \in N$, the following statements are equivalent:

(a) $R_{A}\left(I^{s}\right)$ is a Cohen-Macaulay ring.

(b) $R_{A}\left(\mathbf{I}_{s}\right)$ is a Cohen-Macaulay ring.

Observe that the assertion of the above theorem holds trivially without any additional assumptions if depth $G=d$ (i.e. $G$ is Cohen-Macaulay), since in this case $R$ is Cohen-Macaulay by Lemma 2.3 (a) and [TI, 1.1].

Proof. We only have to show the implication from (a) to (b) and may assume that $\operatorname{depth} G=d-1$ (cp. the above remark). From Theorem 1.2 we know that some multiRees algebra is Cohen-Macaulay. By Theorem 1.1 we then get that $a_{d-1}$ and $a(G)$ are negative (hence so is $e_{d-1}$ ) and that $R_{A}\left(\mathbf{I}_{r}\right)$ is Cohen-Macaulay for all $r \geq-e_{d-1}$, but for no $r<-e_{d-1}$. Moreover, we get from Theorem 1.1 that:

$$
\left[H_{M}^{d}(R)\right]_{n}=(0) \text { for } n \notin\left\{e_{d-1}+1, \ldots,-1\right\} .
$$

This implies that $R_{A}\left(I^{r}\right)$ is also Cohen-Macaulay for all $r \geq-e_{d-1}$ (see the arguments given after Theorem 1.1). In order to prove the theorem, we have to show that $R_{A}\left(I^{r}\right)$ is not Cohen-Macaulay for any $r<-e_{d-1}$. From sequence $(S 2)$ and Lemma 2.9 we get the following sequences:

$$
0 \longrightarrow\left[H_{M}^{d-1}(G)\right]_{n} \stackrel{\varphi_{n}}{\longrightarrow}\left[H_{M}^{d}\left(R_{+}\right)\right]_{n+1} \stackrel{\psi_{n}}{\longrightarrow}\left[H_{M}^{d}(R)\right]_{n} \longrightarrow\left[H_{M}^{d}(G)\right]_{n}
$$

We may exclude the case $e_{d-1}=-1$ since this corresponds by Theorem 1.1 to the trivial case that $R$ is Cohen-Macaulay. Suppose now that $e_{d-1}=-2$. Then the injective map $\varphi_{-2}$ implies that $\left[H_{M}^{d}(R)\right]_{-1} \neq(0)$. This shows the assertion of the theorem under assumption (iv).

To prove the theorem under any of the other assumptions, we may assume from now on that $e_{d-1} \leq-3$. To see that $R_{A}\left(I^{r}\right)$ is not Cohen-Macaulay for any $r<-e_{d-1}$, 
it is enough to show that the following holds:

$$
\left[H_{M}^{d}(R)\right]_{n} \neq(0) \quad \text { for each } n=e_{d-1}+1, \ldots,-2 .
$$

Assume now that condition ( $v$ ) holds. Then (5) follows simply from the injectivity of $\varphi_{n}$. If condition (i) holds, we first have to observe that $\left[H_{M}^{d}(R)\right]_{e_{d-1}+1} \neq(0)$, which follows from the injectivity of $\varphi_{e_{d-1}}$. Now the surjectivity of $\psi_{n}$ for $e_{d-1}<n$ implies (5). From Lemma 3.1 we will see that condition (ii) is just a special case of condition (iii), which remains to be proven. If $a_{d-1} \geq-3$, we can again simply make use of the injectivity of $\varphi_{n}$ to see that (5) holds. For $a_{d-1}<-3$ we only obtain with this argumentation that: $\left[H_{M}^{d}(R)\right]_{n} \neq(0)$ for each $n=e_{d-1}+1, \ldots, a_{d-1}+1$. But what is missing for (5) to hold follows from Lemma 2.10 since depth $A=d-1$ by Lemma 2.11 .

REMARK 2.13. If anyone of the Rees algebras in Theorem 2.12 is Cohen-Macaulay for some $s>0$, we can choose $s:=-e_{d-1}$ to obtain that $R_{A}\left(I^{s}\right)$ and $R_{A}\left(\mathbf{I}_{s}\right)$ are CohenMacaulay and that this $s$ is the smallest integer with this property, because depth $G$ $\geq d-1$ and $\left[H_{M}^{d-1}(G)\right]_{n}=(0)$ for all $n<e_{d-1}$.

The following example shows that the condition $a(G) \leq e_{d-1}$ of the above theorem e.g. holds for form rings of parameter ideals in Buchsbaum local rings. And Lemma 3.1 of the next section in particular tells us that the conditions $a(G) \leq e_{d-1}$ or $a(G) \leq a_{d-1}$ of Theorem 2.12 always imply (under the assumption depth $G \geq d-1$ ) that $(A, \mathfrak{m})$ is not Cohen-Macaulay.

Furthermore, the following example will show that we cannot expect $R$ to be Cohen-Macaulay if we already know that $R_{A}\left(I^{S}\right)$ or even $R_{A}\left(\mathbf{I}_{r}\right)$ is Cohen-Macaulay. In particular, in Theorem 1.2 the multi-Rees algebra cannot be replaced by the Rees algebra in general. This also illustrates Theorem 2.12 .

EXAMPLe 2.14. Let $A$ be a Buchsbaum local $\operatorname{ring}$ with $\operatorname{dim} A=d>0$ and $\operatorname{depth} A=d-1$. (E.g. [SV, Thm. 3] and [Go1, 1.2] guarantee the existence of such a ring $A$ ).

Now let $I$ be a parameter ideal of $A$. Then we get from [Go2]:

$$
\begin{aligned}
& {\left[H_{M}^{i}(G)\right]_{n}=\left\{\begin{array}{ll}
H_{\mathfrak{m}}^{i}(A) & \text { for } n=-i \\
(0) & \text { for } n \neq-i
\end{array} \text { for all } 0 \leq i<d\right.} \\
& \text { and } a(G) \leq-d .
\end{aligned}
$$

(This description of the local cohomology of $G$ does not depend on the condition $\operatorname{depth} A=d-1$.) So we have $a_{0}, \ldots, a_{d-2}=-\infty, a_{d-1}=e_{d-1}=1-d$ and $a(G)<0$. If we choose $d \geq 3$, then $R$ is not Cohen-Macaulay by [TI, 1.1] but $R_{A}\left(\mathbf{I}_{d-1}\right)$ is CohenMacaulay by Theorem 1.1 .

The smallest integer $r$ such that $R_{A}\left(\mathbf{I}_{r}\right)$ is Cohen-Macaulay is equal to $d-1$ (also by Theorem 1.1). Furthermore, we get that $R_{A}\left(I^{s}\right)$ is Cohen-Macaulay for all $s \geq d-1$ 
and Theorem 2.12 tells us that $s:=d-1$ is the smallest number such that $R_{A}\left(I^{s}\right)$ is Cohen-Macaulay.

We close this section with an observation which is related to question II of the introduction. Question II could also be formulated as: When does it follow that the Cohen-Macaulayness of $R_{A}\left(\mathbf{I}_{r+1}\right)$ implies the same property for $R_{A}\left(\mathbf{I}_{r}\right)$ ? The next proposition, though easy to prove, is an interesting observation in this direction. (Necessary and sufficient conditions for the implication in the above question can be found in $[\mathbf{H H K}]$.)

Proposition 2.15. Suppose that $R_{A}\left(I^{s}\right)$ is Cohen-Macaulay for some $s>0$. If $R_{A}\left(\mathbf{I}_{s m+1}\right)$ is Cohen-Macaulay for some $m>0$, then $R_{A}\left(\mathbf{I}_{s m}\right)$ is also Cohen-Macaulay.

Proof. Since $R_{A}\left(\mathbf{I}_{s m+1}\right)$ is Cohen-Macaulay, we get from Theorem 1.1 (b) that $e_{M}^{i}(R) \geq-s m$ for all $i \leq d$. But from the proof of Lemma 2.3 we know:

$$
\left[H_{M}^{i}(R)\right]_{s \cdot n}=(0) \text { for all } n \in Z \text { and } i \leq d,
$$

which in particular holds for $n=m$. Both together imply: $e_{M}^{i}(R) \geq-s m+1$ for all $i \leq d$. Again by Theorem 1.1 (b) this shows that $R_{A}\left(\mathbf{I}_{s m}\right)$ is Cohen-Macaulay.

\section{Proof of Theorem 1.4 and 1.5 .}

Throughout this section, we will assume that $A$ is a Cohen-Macaulay local ring with infinite residue field $A / \mathrm{m}$. In order to prove Theorem 1.4 and 1.5 , one can achieve this situation by passing from $A$ to the ring $A[X]_{\mathfrak{m} A[X]}$ where $X$ is an indeterminate over $A$. Let $J$ be a minimal reduction of $I$, that is a subideal of $I$ generated by $\ell(I)$-many elements and satisfying $I^{n+1}=J I^{n}$ for some $n>0$ (cf. [NR]). Here, $\ell(I)$ denotes the analytic spread of $I$, that is: $\ell(I):=\operatorname{dim} G \otimes_{A} A / \mathrm{m}$. In particular, $\ell(I)=0$ implies that $I$ is nilpotent. It is well known that: $\mathrm{ht}_{A}(I) \leq \ell(I) \leq \operatorname{dim} A$. Let $r_{J}(I)$ stand for the reduction exponent of $I$ with respect to $J$, that is: $r_{J}(I):=\min \left\{n>0 \mid I^{n+1}=J I^{n}\right\}$.

The next lemma is taken from [Ko] and provides us with a generalization of a result of Marley (cf. [Ma, 2.1]), who proved the same for m-primary ideals with a different method. The authors were informed by E. Hyry that the lemma is also shown in a preprint of Hoa (see Theorem 5.2 in [Ho2]). The lemma will play a key role in the sequel.

Lemma 3.1. Let $A$ be Cohen-Macaulay and $\ell(I)>0$. If $g:=\operatorname{depth} G<d$, then: $a_{g}<a_{g+1}$.

If $\operatorname{ht}(I)>0$, the lemma also holds under the weaker assumption: $\operatorname{depth} A>$ $\operatorname{depth} G$ (cp. [Ko]). We also note that for $i<g$ we have by the description of depth via local cohomology: $a_{i}=-\infty$.

Proof. Choose an integer $n>a_{g+1}$. Then we get from sequence $(S 1)$ the inclusion $0 \longrightarrow\left[H_{M}^{g+1}\left(R_{+}\right)\right]_{n} \longrightarrow\left[H_{M}^{g+1}(R)\right]_{n}$ and from sequence $(S 2)$ the surjective 
$\operatorname{map}\left[H_{M}^{g+1}\left(R_{+}\right)\right]_{n+1} \longrightarrow\left[H_{M}^{g+1}(R)\right]_{n} \longrightarrow 0$. Hence we have: $\left[H_{M}^{g+1}\left(R_{+}\right)\right]_{n}=(0)$ for all $n>a_{g+1}$ by the Artinian property of $H_{M}^{g+1}\left(R_{+}\right)$. By $[\mathbf{H M}, 3.10]$ we know: depth $R=\operatorname{depth} G+1$, since $\operatorname{depth} A>\operatorname{depth} G$. (The proof of this in $[\mathbf{H M}$ needs $\operatorname{dim} R=d+1$ which is guaranteed by our assumption $\ell(I)>0$ since $A$ is equidimensional.) So we get from sequence $(S 2): 0 \longrightarrow\left[H_{M}^{g}(G)\right]_{n-1} \longrightarrow\left[H_{M}^{g+1}\left(R_{+}\right)\right]_{n}$ for all $n \in Z$. Together with the above, this yields: $\left[H_{M}^{g}(G)\right]_{n-1}=(0)$ for all $n>a_{g+1}$. This implies the statement of the lemma.

As a first application of the lemma, we obtain the following corollary which adds one more point to the equivalences of Remark 2.7 (under the assumption that $A$ is Cohen-Macaulay):

Corollary 3.2. Let $A$ be a 1-dimensional Cohen-Macaulay ring and $I$ an $\mathrm{m}-$ primary ideal. If $a(G)<0$, then $R$ is a Cohen-Macaulay ring and $I$ is a principal ideal.

ProOf. From Lemma 3.1, the first assertion follows since we have in general: $a_{0} \geq 0$ unless $G$ is Cohen-Macaulay. That $I$ is principal follows from [HIO, (45.4)].

We need one more lemma in the case that $\operatorname{dim} A=1$.

Lemma 3.3. Let I be an ideal in 1-dimensional Cohen-Macaulay local ring $A$ such that $A / I$ is a 1-dimensional Cohen-Macaulay ring. If $a(G) \leq 1$, then $G$ is CohenMacaulay.

Proof. We first assume: $\ell(I)=1$. Look at the long exact sequence of local cohomology coming from $0 \longrightarrow G_{+} \longrightarrow G \longrightarrow A / I \longrightarrow 0$. Then we get an isomorphism $H_{M}^{0}\left(G_{+}\right) \cong H_{M}^{0}(G)$ since $\operatorname{depth} A / I>0$. Now $a(G) \leq 1$, hence $a_{0}(G) \leq 0$ by Lemma 3.1, while $H_{M}^{0}\left(G_{+}\right)$is concentrated in positive degrees. Thus $H_{M}^{0}(G)=(0)$ and hence $G$ is Cohen-Macaulay.

Next we consider the case that $\ell(I)=0$. Then $I$ is nilpotent. Put $r:=$ $\max \left\{n \in Z \mid I^{n} \neq(0)\right\}$. Then it follows by $[\mathbf{G H}, 2.2]$ that $\left[H_{M}^{i}(G)\right]_{r} \cong H_{\mathfrak{m}}^{i}\left(I^{r}\right)$, while $H_{\mathrm{m}}^{0}\left(I^{r}\right)=(0)$ and $a(G) \leq 1$. Hence $r \leq 1$, i.e. $G \cong A / I \otimes I$. Let $x$ be a system of parameters of $A$. Then $x$ is $A / I$-regular and $I$-regular (Note that $A / I$ and $I$ are CohenMacaulay $A$-modules of dimension 1.) Thus $x$ is $G$-regular, which implies that $G$ is Cohen-Macaulay. Hence the lemma follows.

Proposition 3.4. Let $A$ be Cohen-Macaulay of $\operatorname{dim} A \geq 2$. Suppose that $\mathrm{ht}_{A}(I) \geq$ $d-1$. Assume moreover that $\operatorname{depth} G \geq d-1$ and $a(G) \leq 1-d$. Then $G$ is CohenMacaulay.

Proof. Because $A / m$ is infinite, we can choose a superficial element $a \in I$ (see e.g. $[\mathrm{Na}, \S 22])$ and by denoting the image of at in $G$ by $z$, we have $\left[0:_{G} z\right]_{n}=(0)$ for $n \gg 0$. Now look at the following two sequences:

$$
\begin{gathered}
0 \longrightarrow(0: G z)(-1) \longrightarrow G(-1) \longrightarrow z G \longrightarrow 0 \\
0 \longrightarrow z G \longrightarrow G \longrightarrow G / z G \longrightarrow 0
\end{gathered}
$$


Since depth $G>0$, we have:

$$
H_{M}^{0}\left(0:_{G} z\right)=H_{M}^{0}(z G)=(0)
$$

by the above sequences.

We will first show the case $d=2$ and $\operatorname{depth} G \geq 1$. We know by Lemma 3.1 that $a_{1}<a(G) \leq-1$. On the other hand, we have by [GH, 2.2]:

$$
H_{M}^{i}\left(0:_{G} z\right)=\bigoplus_{n \geq 0} H_{\mathfrak{m}}^{i}\left(\left[0:_{G} z\right]_{n}\right) \quad \text { for all } i
$$

Combining both, we see from $0 \longrightarrow H_{M}^{1}\left(0:_{G} z\right) \longrightarrow H_{M}^{1}(G)$ (which follows from sequence (6) and (8)), that $H_{M}^{1}\left(0:_{G} z\right)=(0)$. But from (9), we also get $H_{M}^{2}\left(0:_{G} z\right)=(0)$ since $\left[0:{ }_{G} z\right]_{n} \subseteq I^{n} / I^{n+1} \subseteq A / I^{n+1}$ and $\operatorname{dim} A / I^{n+1} \leq 1$. Hence $z$ is a non-zero divisor on $G$. From the sequence:

$$
0 \longrightarrow G(-1) \stackrel{\cdot z}{\longrightarrow} G \longrightarrow G / z G \longrightarrow 0
$$

we get:

$$
0 \longrightarrow H_{M}^{0}(G / z G) \longrightarrow H_{M}^{1}(G)(-1)
$$

We have already seen that $a_{1} \leq-2$. Therefore, we can deduce from the above injective map: $H_{M}^{0}(G / z G)=(0)$. But this means depth $G / z G \geq 1$ and therefore: $\operatorname{depth} G=2$. This was to show.

Now suppose $d \geq 3$. Using (9) and $\operatorname{dim}_{A}\left(\left[0:_{G} z\right]_{n}\right) \leq \operatorname{dim} A / I^{n+1} \leq 1$ we see: $H_{M}^{i}\left(0:_{G} z\right)=(0)$ for $i \geq 2$. But since $\operatorname{depth} z G \geq 1$ by (8) and $\operatorname{depth} G \geq 2$ we get from sequence (6): $\operatorname{depth}\left(0:_{G} z\right) \geq 2$. Hence $0:_{G} z=(0)$, i.e. $z$ is a non-zero divisor on $G$. Furthermore, $a(G / z G)=a(G)+1$, which follows from the sequence:

$$
H_{M}^{d-1}(G) \longrightarrow H_{M}^{d-1}(G / z G) \longrightarrow H_{M}^{d}(G)(1)
$$

and the fact that: $a_{d-1}<a(G)(\mathrm{cp}$. Lemma 3.1). We now see, that we can reduce to the case $d=2$.

ProOF OF THEOREM 1.4. (b) $\Rightarrow$ (a) follows from Theorem 1.1. (a) $\Rightarrow(\mathrm{b})$ : By Theorem 1.1 we have: $\operatorname{depth} G>0 \quad$ (since always $a_{0} \geq 0$ ) and $a(G)<0$. Hence the assertion immediately follows from Proposition 3.4.

Lemma 3.5. Suppose that $\mathrm{ht}_{A}(I)>0$. Then there exists an element $a \in I$ such that the image $z$ of at $\in R$ in $G$ fulfills the following two conditions:

(a) $\left[(0):{ }_{G} z\right]_{n}=(0)$ for all $n \gg 0$.

(b) $z / 1$ is part of a system of parameters of $G_{\mathfrak{p}}=G_{A_{\mathfrak{p}}}\left(I_{\mathfrak{p}}\right)$ for all $\mathfrak{p} \in \operatorname{Min}_{A}(A / I)$.

Proof. Put $\mathscr{F}:=\operatorname{Ass}(G) \backslash V\left(G_{+}\right)$and $\mathscr{G}_{\mathfrak{p}}:=\left\{Q \in \operatorname{Spec}(G) \mid Q \otimes A_{\mathfrak{p}} \in \operatorname{Assh}_{G_{\mathfrak{p}}}\left(G_{\mathfrak{p}}\right)\right\}$ for each $\mathfrak{p} \in \operatorname{Min}_{A}(A / I)$. Then $\mathscr{H}:=\mathscr{F} \cup\left(\bigcup_{\mathfrak{p} \in \operatorname{Min}(A / I)} \mathscr{G}_{\mathfrak{p}}\right)$ is a finite set. Since $\operatorname{dim} G_{\mathfrak{p}}>0$ and $\operatorname{dim}\left(G_{0}\right)_{\mathfrak{p}}=0$ for all $\mathfrak{p} \in \operatorname{Min}_{A}(A / I), G_{1}$ cannot be contained in any $Q \in \mathscr{G}_{\mathfrak{p}}$. And obviously, $G_{1} \nsubseteq Q$ for all $Q \in \mathscr{F}$. Since $A / \mathrm{m}$ is infinite, we can thus 
choose $a \in I$ such that $z \in G_{1} \backslash \bigcup_{Q \in \mathscr{H}} Q$, where $z$ denotes the image of at $\in R$ in $G$. Then $z$ satisfies (b) of the lemma. (a) also holds since $z$ avoids by its choice all prime ideals in $\mathscr{F}$ and is therefore a superficial element in $G$ (cp. e.g. [Na, (22.1)] or [Tr]).

Proof of TheOrem 1.5. It is enough to show the implication $(\mathrm{a}) \Rightarrow(\mathrm{b})$. Suppose that $R_{A}\left(\mathbf{I}_{r}\right)$ is Cohen-Macaulay. By [TI, 1.1] and the fact that $a(G)<0$ which follows from Theorem 1.1, it is enough to see the Cohen-Macaulayness of $G$.

We will consider first the case of an integrally closed m-primary ideal $I$ in $A$. Let $J$ be a minimal reduction of $I$. By [Tr, 3.2] we have:

$$
r_{J}(I) \leq \max \left\{a_{i}(G)+i \mid 0 \leq i \leq 3\right\},
$$

while Theorem 1.1 (and the above) tells us $a_{i}(G)<0$ for $i \leq 3$. Thus we have $I^{3}=J I^{2}$, and it follows that $I^{n+1} \cap J=J I^{n}$ for all $n \geq 2$. On the other hand, by [It, 1.1], we have $\overline{J^{2}} \cap J=\bar{J} J$ since $J$ is generated by an $A$-regular sequence. Here $\bar{J}$ denotes the integral closure of $J$. Since $I=\bar{J}$ and $I^{2} \subseteq \overline{J^{2}}$, we get $I^{2} \cap J=J I$. Hence by [VV, 2.7], $G$ is a Cohen-Macaulay ring.

Now we take an ideal $I$ so that $A / I$ is a 1-dimensional Cohen-Macaulay ring. Choose $a \in I$ such that the image $z$ of $a t$ in $G$ is an element as in Lemma 3.5. Let $Q \in \operatorname{Ass}_{G}((0): G z)$. Then $Q$ is a homogeneous ideal and $G_{+} \subseteq Q$. Let $\mathfrak{p}=Q \cap A$. Then $I \subseteq \mathfrak{p} \subseteq \mathfrak{m}$.

If $\mathfrak{p} \neq \mathfrak{m}$, then $\mathfrak{p} \in \operatorname{Min}_{A}(A / I)$ and $A_{\mathfrak{p}}$ is a 2-dimensional Cohen-Macaulay ring. Hence we get that $G_{A_{\mathfrak{p}}}\left(I_{\mathfrak{p}}\right)$ is a Cohen-Macaulay ring by Theorem 1.4. (Note that $R_{A}\left(\mathbf{I}_{r}\right) \otimes_{A} A_{\mathfrak{p}}$ is Cohen-Macaulay.) Hence $z / 1$ is $G_{A_{\mathfrak{p}}}\left(I_{\mathfrak{p}}\right)$-regular by Lemma 3.5 and therefore: $\left((0):_{G} z\right)_{\mathfrak{p}}=(0)$. But this is a contradiction.

So we have $\mathfrak{p}=\mathfrak{m}$, i.e. $\operatorname{Supp}_{G}\left((0):_{G} z\right) \subseteq\{M G\}$. But we know from Theorem 1.1 that depth $G>0$. So we get that $z$ is $G$-regular.

Let $B=A / a A$. Then we have an isomorphism $G / z G \cong G_{B}(I B)$ by $[\mathrm{VV}, 2.1]$. Furthermore, again by Theorem 1.1, $a_{i}(G)<0$ for all $i \leq 3$. Thus we get $a_{i}\left(G_{B}(I B)\right) \leq 0$ for $i \leq 2$. Hence it follows from Lemma 3.1 that depth $G_{B}(I B)>$ 0 . We choose an element $b \in I$ once more in such a way that the image $w$ of $b t$ in $G_{B}(I B)$ is an element as in Lemma 3.5. Let $Q \in \operatorname{Ass}_{G_{B}(I B)}\left((0):_{G_{B}(I B)} w\right)$ and $\mathfrak{p}=Q \cap B$. Then $\mathfrak{p}=\mathfrak{m} B$. In fact, if $\mathfrak{p} \neq \mathfrak{m} B$, then $G_{B_{\mathfrak{p}}}\left(I B_{\mathfrak{p}}\right)$ is Cohen-Macaulay (Note the isomorphism $\left.G / z G \cong G_{B}(I B)\right)$. Thus $w$ is $G_{B_{\mathfrak{p}}}\left(I B_{\mathfrak{p}}\right)$-regular, which contradicts the choice of $Q$. Therefore we get $\mathfrak{p}=\mathfrak{m} B$ and $Q=m G_{B}(I B)+G_{B}(I B)_{+}$. Hence $w$ is a $G_{B}(I B)$-regular element because depth $G_{B}(I B)>0$.

Let $C=A /(a, b) A$. Then $C$ is a 1-dimensional Cohen-Macaulay ring, $\mathrm{ht}_{C}(I C)=0$, $a_{i}\left(G_{C}(I C)\right) \leq 1$ and $C / I C(=A / I)$ is Cohen-Macaulay. Hence, by Lemma 3.3, $G_{C}(I C)$ is Cohen-Macaulay. Now, since $z$ and $w$ form a $G$-regular sequence and $G /(z, w) G \cong G_{C}(I C)$, we get that $G$ is a Cohen-Macaulay ring. Hence, by [TI, 1.1], $R$ is Cohen-Macaulay as claimed.

AcKnowledgements. Parts of this work stem from the thesis of the first author which was partially prepared when he was visiting Japan. He wants to thank Professor 
Goto and Professor Ishikawa for the hospitality he received during his stay at Meiji and Tokyo Metropolitan University and all the members of the Meiji-seminar on Commutative Algebra for stimulating discussions. For financial support in this time he wants to thank the JGCB (Japanese German Center Berlin).

This paper was written when the second author visited Germany. He is greatly indebted to Professor Manfred Herrmann and the members of his workgroup on Commutative Algebra at the University of Cologne for their hospitality and stimulating discussions. For financial support he wants to thank the Heinrich-Hertz-Stiftung (Germany).

\section{References}

[Gol] S. Goto, On Buchsbaum rings. J. of Algebra 67, No. 2 (1980), 272-279.

[Go2] S. Goto, On the associated graded rings of parameter ideals in Buchsbaum rings. J. of Algebra, 85, No. 2 (1983), 490-534.

[GH] S. Goto and S. Huckaba, On graded rings associated to analytic deviation one ideals. Amer. J. Math. 116 (1994), 905-919.

[GN] S. Goto and K. Nishida, The Cohen-Macaulay and Gorenstein Rees algebras associated to filtrations. Mem. Amer. Math. Soc. 526 (1994).

[GW] S. Goto and K. Watanabe, On graded rings, I. J. Math. Soc. Japan 30 (1978), 179-213.

[HHK] M. Herrmann, E. Hyry and T. Korb, Filter-regularity and Cohen-Macaulay multi-graded Rees algebras. (in preparation).

[HHR1] M. Herrmann, E. Hyry and J. Ribbe, On the Cohen-Macaulay and Gorenstein properties of multi-Rees algebras. Manus. Mat. 79 (1993), 343-377.

[HHR2] M. Herrmann, E. Hyry and J. Ribbe, On multi-Rees algebras (with an appendix by N. V. Trung). (To appear in Math. Ann.).

[HHRTa] M. Herrmann, E. Hyry, J. Ribbe and Z. Tang, Reduction numbers and multiplicities of multigraded structures. (preprint).

[HIO] M. Herrmann, S. Ikeda, and U. Orbanz, Equimultiplicity and Blowing Up (with an appendix by B. Moonen). Springer-Verlag, Berlin-Heidelberg-New York (1988).

[HRS] M. Herrmann, J. Ribbe and P. Schenzel, On the Gorenstein property of form rings. Math. Z. 213 (1993), 301-309.

[HRZ] M. Herrmann, J. Ribbe and S. Zarzuela, On the Gorenstein property of Rees and form rings of power of ideals. Trans. Amer. Math. Soc. 342, No. 2 (1994), 631-643.

[Hol] L. T. Hoa, Reduction numbers and Rees algebras of powers of an ideal. Proc. of the AMS 119, No. 2 (1993), 415-422.

[Ho2] L. T. Hoa, Reduction numbers of equimultiple ideals. (preprint).

[HZ] L. T. Hoa and S. Zarzuela, Reduction number and a-invariant of good filtrations. (preprint).

[HM] S. Huckaba and T. Marley, Depth formulas of certain graded rings associated to an ideal. Nagoya Math. J. 133 (1994), 57-69.

$[\mathrm{Hu}]$ C. Huneke, On the associated graded ring of an ideal. Illinois J. Math. 26, No. 1 (1982), 121137.

[Ik] S. Ikeda, On the Gorensteinness of Rees algebras over local rings. Nagoya Math. J. 102 (1986), 135-154.

[It] S. Itoh, Integral closure of ideals generated by regular sequences. J. of Algebra 117 (1988), 390401.

[Ko] T. Korb, On $a$-invariants, filter-regularity and the Cohen-Macaulayness of graded algebras. Thesis (1995), University of Cologne.

[Ma] T. Marley, The reduction number of an ideal and the local cohomology of the associated graded ring. Proc. of the AMS 117, No. 2 (1993), 335-341.

[Na] M. Nagata, Local rings. Interscience (1962).

[NR] D. G. Northcott and D. Rees, Reductions of ideals in local rings. Proc. Camb. Phil. Soc. 50 (1954), 145-158. 
[Oo] A. Ooishi, On the Gorenstein property of the associated graded ring and the Rees algebra of an ideal. J. of Algebra 155 (1993), 397-414.

[Re] D. Rees, Generalizations of reductions and mixed multiplicities. J. London Math. Soc. (2) 29 (1984), 397-414.

[Sa] J. D. Sally, On the number of generators of powers of an ideal. Proc. of the AMS. 53 (1975), 24-26.

[SV] J. Stückrad and W. Vogel, Toward a theory of Buchsbaum singularities. Amer. J. Math. 100 (1978), 727-746.

[Te] B. Teissier, Cycles évanescents, sections planes et conditions de Whitney. Singularitiés à Cargese 1972, Astérisque 7-8 (1973), 285-362.

[Tr] N. V. Trung, Reduction exponent and degree bound for the defining equations of graded rings. Proc. of the AMS 101, No. 2 (1987), 229-236.

[TI] N. V. Trung and S. Ikeda, When is the Rees algebra Cohen-Macaulay? Communications in Algebra 17 [12] (1989), 2893-2922.

[VV] P. Valabrega and G. Valla, Form rings and regular sequences. Nagoya Math. J. 72 (1978), 93101.

[Ve] J. K. Verma, Joint reductions and Rees algebras. Math. Proc. Camb. Phil. Soc. 109 (1991), $335-342$.

\section{Thomas KORB}

Mathematisches Institut der Universität zu Köln Weyertal $86-90$ 50931 Köln [Germany] e-mail: korb@mi.uni-koeln.de

\section{Yukio NAKAMURA}

Department of Mathematics Tokyo Metropolitan University Minami-Ohsawa 1-1 Hachioji-shi, Tokyo 192-03 [Japan] e-mail: ynakamu@math.metro-u.ac.jp 